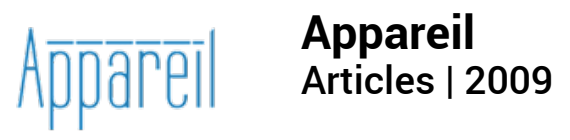

\title{
La pratique musicale comme contre-conduite chez Fela Kuti
}

Julien Perez

\section{(2) OpenEdition}

Journals

Édition électronique

URL : http://journals.openedition.org/appareil/802

DOI : 10.4000/appareil.802

ISSN : 2101-0714

Éditeur

MSH Paris Nord

\section{Référence électronique}

Julien Perez, «La pratique musicale comme contre-conduite chez Fela Kuti », Appareil [En ligne], Articles, mis en ligne le 30 avril 2009, consulté le 10 décembre 2020. URL : http:// journals.openedition.org/appareil/802 ; DOI : https://doi.org/10.4000/appareil.802

Ce document a été généré automatiquement le 10 décembre 2020.

\section{(c) (i) (9)}

Appareil est mis à disposition selon les termes de la Licence Creative Commons Attribution - Pas d'Utilisation Commerciale - Pas de Modification 4.0 International. 


\title{
La pratique musicale comme contre- conduite chez Fela Kuti
}

\author{
Julien Perez
}

1 À l'orée des années soixante-dix, le musicien nigérian Fela Kuti forge un style musical qu'il baptise l'Afrobeat, mélange de chansons et d'instruments du terroir au highlife ghanéen, avec des influences rhythm'n'blues, soul, jazz et funk venues d'Amérique. En 1977, il n'a de cesse de propager son hymne, Zombie, chanson dont le destin conditionne l'extradition de Fela à la fin de cette même année. Nous nous proposons de revenir sur cette décennie au cours de laquelle Fela tente de créer une rupture, à la fois artistique et politique, à partir du champ musical. Plus précisément, l'art et le politique, ces deux sphères que l'on juge volontiers hétérogènes, paraissent ici enchevêtrés à un point qui force l'interrogation de leurs rapports. Notre ambition n'est pas de faire émerger à l'aune de cette étude des connexions objectivantes entre musique et politique mais plutôt de comprendre comment un musicien peut formuler, envisager et vivre ces rapports possibles.

2 Il nous semble également intéressant de faire dialoguer les faits et gestes de Fela en cette période avec l'élaboration conceptuelle des notions de résistance et de contreconduite chez Foucault. Cet intérêt réside notamment en ce que le contexte dans lequel Fela tente de renverser certains rapports de domination est éloigné de celui, principalement occidental, à partir duquel Foucault échafaude sa réflexion, bien que comportant des traits de ressemblance, particulièrement à travers des formes de gouvernementalité telles que le pastorat ou la raison d'État. En effet, le Nigéria durant cette période est un pays aux mains de l'armée. Le pays est dirigé par des militaires dès 1966, soit trois ans après s'être constitué comme une république démocratique. Dès l'indépendance acquise en 1963 éclatent des guerres ethniques qui enlèvent rapidement toute légitimité de représentation au gouvernement constitutionnel. L'armée accède alors au pouvoir pour rétablir l'ordre, justifiant son ingérence dans la sphère politique par l'argument démocratique suivant lequel le gouvernement élu n'est plus apte à représenter et à protéger les intérêts de la nation. Cohabitent alors de manière paradoxale le modèle d'une société militaire calqué sur la relation univoque 
qui lie l'État-major à son armée et le modèle d'une société démocratique où un espace de discussion public est censé assurer une dynamique de l'équivocité selon laquelle les impulsions du peuple sont reprises par le gouvernement dans un mouvement perpétuel. Du fait de cette situation, la contestation embrasse souvent à la fois l'autoritarisme militaire et la légitimité de la représentation démocratique.

3 Alors que Foucault analyse l'émergence de sociétés occidentales modernes dans lesquelles le contrôle et la gestion de la population passent davantage par une homogénéisation et un conditionnement diffus que par la coercition, le Nigéria des années soixante-dix est un pays où la violence d'État est omniprésente. À ce titre, on ne peut qu'être frappé par l'abîme qui sépare Fela d'individus occidentaux étant à la même époque dans une position de résistance, comme par exemple les acteurs du mouvement punk. Si la musique peut être contestataire en Occident, elle met rarement en jeu l'intégrité physique des artistes. À l'inverse les grèves de la faim, les attentats suicide ou les immolations en guise de protestation interfèrent peu avec des considérations artistiques. Or l'avènement de Fela comme grand musicien se déploie dans une proximité déconcertante avec la mort et la destruction. Des paroles de Zombie décrivant la cruauté de l'armée nigériane au dépôt du corps de la mère de Fela devant la résidence du général Obasanjo semblent s'élaborer des stratégies de résistance qui, en l'absence d'un espace de dissensus sauf, sont infiniment moins discursives que les tentatives occidentales contemporaines. Ces stratégies se déploient plutôt dans ce champ de bataille que constitue le corps dont le statut oscille constamment entre celui de véhicule d'aliénation, d'humiliation, d'anéantissement et celui de transe, d'instrument polyvalent d'action et de création, moyen de libération individuelle et collective. Il n'en fut pas toujours ainsi dans la vie de Fela et c'est pour cela que nous nous limitons à cette période. Quelques années plus tard, dans un climat politique apaisé, il donnera à sa lutte une forme plus argumentée et conventionnelle en se présentant aux élections présidentielles, mais l'année 1977 nous semble plus intéressante en ce que la musique de Fela, dans sa pratique et sa réception, est alors incitation à se conduire différemment face à la mort.

4 L'Afrobeat affiche différentes ambitions contenues dans la polysémie de son nom: battement cardiaque, rythme, combat.

5 Fela pose les bases de ce style musical au Nigéria en 1963, mais cette appellation désigne alors moins la naissance d'une forme artistique révolutionnaire qu'une tentative pour tenir le choc face à son concurrent Geraldo Pino, qui joue la musique de James Brown et des autres artistes soul américains et dont le succès nigérian est immense. En effet, la musique de Fela est à cette époque un mélange de highlife, musique populaire urbaine d'origine ghanéenne occupant alors la majorité du paysage musicale nigérian, et de jazz sans grande portée novatrice, et celui-ci demeure un musicien relativement insignifiant. Quelque temps plus tard, en 1967, les atrocités perpétrées pendant la guerre civile nigériane ayant lieu au Biafra ébranlent son patriotisme mais ne changent pas radicalement son approche de la pratique musicale. En 1969, il fait un voyage aux États-Unis et rencontre Sandra Isidore, une militante de la cause noire américaine. Il découvre alors les écrits du militantisme noir radical comme ceux de Malcolm X. Il devient immédiatement un fervent défenseur du panafricanisme et décide de refonder l'Afrobeat auquel il incorpore des éléments soul, rythm'n'blues et funk américains ainsi que des chants africains traditionnels. La théorisation de l'Afrobeat comme synthèse de toutes les grandes musiques noires 
semble totalement solidaire d'une prise de conscience des humiliations dont la diaspora africaine est victime.

6 L'analyse de la genèse de l'Afrobeat ne semble donc pas autoriser la séparation entre une certaine pression de la modernité artistique qui pousse le musicien à repenser sa pratique, à se réinventer, et la nécessité, quant à certains effets de pouvoir et états de domination extérieurs à la sphère musicale, de situer cette pratique artistique. Elle nous intéresse sous deux aspects. D'une part, elle semble être un matériau propice à dialoguer avec la conceptualisation foucaldienne des contre-conduites et des stratégies de résistance. D'autre part, elle permet de penser certaines intrications entre musique et pouvoir.

7 L'Afrobeat est d'emblée un combat et le premier point de croisement avec les analyses foucaldiennes réside dans le fait que cette arme a des cibles multiples qu'on ne saurait résoudre en un pôle de pouvoir situé et homogène. Les relations de pouvoir contre lesquelles s'élabore l'Afrobeat ne se limitent pas à un seul type de gouvernement politique ou économique mais s'étendent à de multiples niveaux de la vie sociale et culturelle. Il suffit d'explorer la discographie de Fela dans les années soixante-dix pour rendre compte de cette multiplicité. Avec les morceaux Shakara et Yellow Fever, il dénonce la manière dont les femmes nigérianes s'occidentalisent, par leurs tenues et par l'usage de crèmes blanchissantes. Sur Gentleman, il s'adresse aux hommes africains en leur demandant de ne pas s'habiller à l'occidentale alors que Confusion ou No Bread appellent à une meilleure répartition des ressources, notamment le pétrole nigérian, et fustigent le colonialisme et le néo-colonialisme. Enfin, Zombie marque son entrée en guerre contre le gouvernement militaire nigérian.

8 À l'éclatement de ces cibles répond une multiplication plutôt anarchique des stratégies et tactiques de résistance adoptées par Fela qui interdit la réduction de ce combat à une opposition simpliste entre deux positions stables. Ici encore, les caractéristiques que donne Foucault de la résistance comme n'étant pas «antérieure au pouvoir qu'elle contre " mais «coextensive et absolument contemporaine $»^{1}$ ainsi que son analyse, dans ses cours au Collège de France, des contre-conduites relatives à la pastorale chrétienne qui ne se révèlent pas extérieures au christianisme mais dont les éléments tactiques sont inhérents au régime dont elles tentent de se dissocier ${ }^{2}$ semblent éclairer l'apparente incohérence des choix de Fela.

9 Ce dernier choisit de chanter en anglais pidgin, l'anglais du peuple, au détriment de sa langue maternelle, le yoruba, afin d'être compris par un plus grand nombre d'Africains. Il critique le régime militaire nigérian à partir du concept occidental de droits de l'homme alors qu'il combat violemment l'influence de l'Occident sur le continent africain, allant jusqu'à considérer le sida comme l'une de ses mystifications. Il oppose aux symboles militaires le poing levé des Black Panthers et un nom de chef de guerre, Anikulapo, «celui qui porte la mort dans sa bourse». Il proclame sa grande demeure nigériane "république libre de Kalakuta » et la dote d'un studio d'enregistrement, d'un marché, d'un hôpital et d'une prison. Dans cette liste des quelques mesures phares entreprises par Fela et qui eurent les effets escomptés de déstabilisation des rapports de domination visés, on ne peut que constater l'absence d'extériorité de ces stratégies de résistance vis-à-vis de ce qu'elles combattent. D'une part, elles lui empruntent souvent les mêmes armes en en modifiant le champ d'application et la portée. D'autre part, elles ne semblent pas aboutir à une libération mais à une modification des rapports, ainsi nous pourrions aussi bien prolonger le travail de Surveiller et punir ou de 
Naissance de la clinique dans l'analyse des prisons et des hôpitaux de la république nigériane des années soixante-dix que dans l'analyse de la prison et de l'hôpital de la « république libre de Kalakuta ».

10 Le deuxième aspect mobilisant notre attention dans la formation de l'Afrobeat vise les relations entre musique et pouvoir. Compte tenu des éléments biographiques que nous venons de citer, on peut en effet se poser la question de savoir si l'engagement de Fela dans sa lutte contre le gouvernement nigérian et l'impérialisme occidental est contingent quant à son apport artistique. On peut en effet penser qu'il existe un devenir militant du musicien, ni plus ni moins que pour quiconque, lorsqu'il juge la nécessité d'agir en vue d'une émancipation quelconque mais que cela ne le prémunit pas d'endurer l'irrémédiable scission entre musique et politique, en comprenant ce dernier terme comme champ d'action permettant d'agir sur l'action des autres. Or, nous soutenons qu'on ne peut effectuer cette scission dans la naissance de l'Afrobeat. Autrement dit, la découverte des écrits du militantisme noir permet à Fela de penser ce style musical révolutionnaire qui, en retour, lui donne une incidence nouvelle sur les rapports de pouvoir qu'il entend renverser. Dans ce cas précis, le musicien et le militant sont indissociables même si le partage peut s'effectuer sur certains faits biographiques. L'Afrobeat n'est pas une musique appliquée, une musique censée soutenir un élément hétérogène en l'esthétisant, comme la musique inaugurant une allocution présidentielle ou les nappes de violons accentuant le pathos d'une scène de cinéma. Il ne s'identifie pas non plus à la pulsation du chant guerrier qui vient donner du courage aux combattants ou exalter leur appartenance à un groupe. Les textes de Fela ne se greffent pas à la musique comme un surplus hétérogène qui aurait pu être tout autre. À ce sujet, il déclare dans un entretien : «It is very difficult for me not to have music that is not politics. Even if it's not politics, it would have to be music that is very culturally aware for people to see the beauty of the African concept. ${ }^{3} \mathrm{Ces}$ mots suggèrent la pratique d'une musique comportant une charge politique qui ne se situe pas dans le caractère engagé des paroles d'une chanson mais dans la forme musicale elle-même. Selon Fela, ce qui doit restaurer la fierté de l'Afrique noire et de ses ressortissants est une pratique musicale censée exacerber la grandeur de leurs facultés sensibles et intellectuelles. L'enjeu consiste à montrer que l'Occident n'a pas le monopole de la virtuosité et de la création de nouvelles formes capables de changer la sensibilité commune. De plus, et ce malgré l'influence de la pensée panafricaine dans la formation de ce style qui pourrait laisser croire que ce dernier répond à un certain phantasme unitaire, il nous semble que par ses origines multiples, la musique Afrobeat possède également quelque chose d'apatride qui marque une volonté d'émancipation artistique vis-à-vis du pouvoir officiel incarné par le gouvernement d'un pays quelconque. Il est à propos de rappeler que la modernité musicale africaine, contrairement à celle ayant cours en Occident, est intimement liée au pouvoir politique. Le highlife, par exemple, dont il était question précédemment, naît au sein de fanfares militaires et d'orchestres accompagnant le cortège officiel du président ghanéen Nkrumah au cours de ses déplacements. L'Afrobeat, par l'éclatement volontaire de ses bases, est structurellement barbare vis-à-vis de n'importe quel territoire étatique et tend à affirmer que les intérêts africains qu'il défend ne sont pas les mêmes que ceux des divers gouvernements dispersés sur le continent.

11 On pourrait dire que l'Afrobeat peut se penser par rapport aux différents nœuds de pouvoir décrits précédemment selon un double mouvement: une distanciation créatrice à partir de laquelle il établit ses propres normes et un mouvement plus 
offensif en ce que ces nouvelles normes remettent en question l'apparente immuabilité des relations de pouvoir selon lesquelles l'art occidental est supérieur à l'art africain et les différentes musiques africaines sont une collection de célébrations étatiques.

Il nous importe maintenant d'examiner plus soigneusement les contre-conduites et les stratégies de résistance qu'oppose Fela au pouvoir qui s'exerce sur lui de la façon la plus directe alors que le général Obasanjo est à la tête du gouvernement nigérian.

En 1977, Fela et son groupe Afrika 70 composent le morceau Zombie à la fois reconnu comme l'une des plus grandes réussites musicales de l'Afrobeat et comme la première charge frontale envers l'armée nigériane. Ce morceau nous paraît particulièrement digne d'intérêt en ce que semble s'enrouler autour de son devenir l'histoire d'une lutte entre différents corps : corps de zombie, corps de danseur, corps de musicien, corps de théâtre, corps de martyr.

Le premier corps, le corps dont la description déclenche le combat, est un corps dépossédé de toute singularité. Il ne possède aucun attribut, il est un corps inactif au sens où il ne saurait être sa propre cause.

Zombie no go go, unless you tell am to go Zombie no go stop, unless you tell am to stop Zombie no go turn, unless you tell am to turn Zombie no go think, unless you tell am to think.Ce corps indifférent est commun aux soldats qui exécutent les ordres et aux civils qui laissent faire sans questionner la légitimité de ces actions. C'est un corps séparé de sa puissance d'agir dont les mouvements sont déterminés par une cause extérieure. Zombie est un mot qui désigne, dans le vaudou, quelqu'un qui est sous le contrôle total d'un sorcier. Après avoir été affectée par une drogue puissante, la victime aurait tous les aspects d'un mort par un arrêt complet apparent des fonctions vitales. Le zombie est donc celui que l'on a privé de son âme, coupé de sa volonté.

Dans Surveiller et punir, Foucault décrit ces corps dociles que fabrique la discipline comme des corps dont les forces sont «majorées en termes économiques d'utilité et diminuées en termes politiques d'obéissance $»^{4}$. Il ne nous parait pas anachronique d'effectuer ce rapprochement entre cette description du «moment historique des disciplines $»^{5}$ que Foucault situe au XVIII ${ }^{e}$ siècle européen et la condition des soldats nigérians dont parle cette chanson car les techniques disciplinaires dont ils sont l'objet sont directement héritées du colonialisme britannique. Il est difficile de ne pas reconnaître ce rapport qui rend le corps humain « d'autant plus obéissant qu'il est plus utile, et inversement $»^{6}$ dans un pays qui fut aux mains de l'armée de 1966 à 1979, les principaux protagonistes se succédant au gré des coups d'État, puis de 1983 à 1999. Cette omniprésence de l'armée dans cette partie de l'histoire nigériane consacre l'efficacité de ses agents et la non remise en cause de sa légitimité. Les soldats décrits par Fela sont d'autant plus efficaces qu'ils sont soumis.

Go and kill - joro, jara, joro Go and die - joro, jara, joro Go and quench- joro, jara, joro

Ces soldats tuent, détruisent et meurent sans que rien ne fasse obstacle à leur funeste labeur. L'assaut de Kalakuta est la triste confirmation de ce constat.

Pour déstabiliser l'efficacité de cette "anatomie politique $»^{7}$ ou "mécanique du pouvoir $\|^{8}$ il semble qu'il faille réactiver ces corps tristement matériels, réduits à n'être qu'agents ou objets de destruction. Le morceau de Fela n'est pas une simple description de cet état d'apathie politique et d'efficacité sanguinaire caractéristique du zombie mais est une véritable entreprise de désenvoûtement véhiculée par la musique. On s'interdirait probablement de comprendre cela en se limitant à l'étude de 
l'enregistrement audio officiel de ce morceau qui conserve une force manifeste mais qui ne peut rendre compte à lui tout seul de ses répercutions à l'extérieur d'une sphère strictement esthétique. Il faut donc rappeler que Fela, dès 1973, possède un club, l'AfroShrine, dans lequel il se produit plusieurs fois par semaine, l'industrie du disque n'étant alors pas assez développée en Afrique pour permettre aux musiciens de vivre de leurs ventes de disques. Il apparaît donc nécessaire d'envisager Zombiedans sa réactualisation constante au cours de concerts marathoniens où se mêlent improvisations chorégraphiques, rythmiques, mélodiques et martèlement de la ritournelle Zombie, oh zombie. Ajoutons également que la musique africaine n'envisage pas l'œuvre musicale comme conçue et fixée par l'écriture mais comme un ensemble formel plus ou moins complexe, plus ou moins défini selon les cas, à partir duquel prend naissance l'œuvre proprement dite au moment où elle se joue. Elle prend donc corps véritablement lorsqu'elle se réalise dans le temps concret, lorsqu'elle se joue. Il est ainsi salutaire de visionner les rares images de ces performances pour sentir ce combat au corps à corps, pour entendre la répétition de ces paroles décrivant l'état du zombie jusqu'à l'essoufflement, en faisant ainsi surgir le caractère absurde et insoutenable, pour approcher cette tentative de réanimation par la transe. En effet, il nous semble que la lutte que constitue ce morceau, et d'une manière plus générale l'Afrobeat, se situe davantage dans une opposition entre le corps envoûté et le corps en transe que dans une volonté de prise de conscience, comprise comme réappropriation de qualités premières ayant fait l'objet d'une confiscation. Le corps n'est pas le dernier bastion de révolte au sens où il serait une citadelle inexpugnable en tant que réalité close et intime. Zombiedit tout le contraire. En revanche, le corps du musicien-danseur a un pouvoir de dématérialisation, une capacité fugitive au sens propre. Au rythme atrophié par la cadence de la marche militaire, enferré dans l'enclos d'une arithmétique binaire auquel Zombie fait référence - quick march, slow march, left turn, right turn - s'oppose une autre forme de répétition. L'Afrobeat est éminemment répétitif. D'abord, il constitue l'une des formes les plus abouties d'un art de la polyrythmie caractéristique des musiques africaines. La polyrythmie est une suite de formules rythmiques plus ou moins irréductibles qui s'organisent par rapport à une pulsation régulière et isochrone, exprimée ou sous-entendue. La répétition est donc traversée de subtiles variations d'accentuation, de résonance, de timbre et de durée, la formule apparemment répétitive est fondamentalement mouvante. Il en va de même pour le chant qui se déploie en répétitions de quelques motifs vocaux sujets à d'infimes variations d'intensité et de répartition chant/contre-chant, chant/chœurs. Enfin, les mouvements répétés des danseurs provoqués par cette forme musicale tendent à provoquer, au moyen d'automatismes musculaires, une sorte d'inconscience. La frénésie rythmique provoquant trépignements, déhanchements, balancements et tournoiements aboutit à l'éclipse de la vie consciente, à la déprise de soi. À l'image du zombie dont la conscience appartient désormais à celui qui l'a envoûté s'oppose donc la transe suscitée par la pratique de la musique et de la danse en ce qu'elle interdit la correspondance entre état d'inconscience et état d'assujettissement. Nicole Sindzingre, dans un article consacré à la possession dans les sociétés traditionnelles, établit une distinction entre transe et possession. Le premier terme met l'accent sur « la dissociation de la personnalité, qui s'accompagne de manifestations telles que l'hypnose ou les automatismes ${ }^{9}$ alors que le second n'entraîne pas forcément la transe, «la maladie, par exemple, étant fréquemment perçue comme un état de possession, la transe pouvant survenir seulement au cours du traitement consécutif à ce diagnostic, notamment lors de 
cérémonies d'exorcisme $»^{10}$. La transe est circonscrite à sa condition de phénomène extatique et correspond à l'état d'un individu inspiré dont l'âme voyage dans l'univers non humain, alors que la possession a davantage le sens d'une évaluation culturelle par les membres du groupe de la condition du possédé. On peut donc envisager le corps du musicien et du danseur en transe comme instance de résistance à la prise de pouvoir d'un agent extérieur. Cela permet peut-être d'éclairer cette description de la pratique musicale par Fela « Music is a spiritual thing. You don't play with music. If you play with music you will die young. See, because when the Higher Forces give you gift of music, musicianship, it must be well used for the good of humanity ${ }^{11}$.

La musique n'est pas simplement source de divertissement, de plaisir, et le moyen possible d'un enrichissement ou d'une reconnaissance. Elle est aussi ce par quoi le corps peut se spiritualiser et acquérir une humanité qui déborde son caractère mécanique et périssable.

21 On pourrait reprendre ces mots d'Alain Badiou au sujet de la danse qui n'est qu'un corps et qui force pourtant à admettre que dans sa pratique complexe et virtuose se joue quelque chose comme un "corps spirituel ou un corps spiritualisé ou un corps allégé de toute pesanteur, un corps à la fois terrestre et supraterrestre $»^{12}$. Il dit du corps du danseur qu'il est «un corps disposé comme pensée dans l'espace, disposé comme spiritualité artistique dans l'espace $»^{13}$. Nous ajouterons dans le cas étudié que cette pensée n'est pas de l'ordre de la maîtrise, mais d'une pensée fluidifiée, en mouvement perpétuel.

« Ne pas jouer avec la musique » c'est à la fois prendre au sérieux sa puissance effective, sa capacité à susciter de la pensée et ne pas la considérer comme le simple fruit d'un apprentissage, la stricte application de techniques et de normes n'ayant qu'une visée affective inoffensive. C'est aussi ne pas la considérer comme un outil, le véhicule d'un discours, ce que suggère le "with ». " Music is the weapon $»^{14}$ conclue Fela, la musique elle-même agit sur les êtres, elle envole la pensée, elle est une force de frappe et une force de résistance.

Cette arme est utilisée par Fela au début de l'année 1977. En réponse au FESTAC (Festival for Black Arts and Culture) organisé par le gouvernement nigérian afin d'asseoir son autorité et de promouvoir les cultures noires venues du monde, il organise pendant la même période un contre-festival dans son fief de Kalakuta afin de marquer son désaccord avec la politique que mène alors Obasanjo, ex-chef des armées, qui vient de remplacer le général Murtala Mohammed assassiné l'année précédente et ayant lui-même pris le pouvoir à la faveur d'un coup d'État en juillet 1975. D'une part, on peut remarquer que le FESTAC, organisé dans une période où cohabite misère économique et haute instabilité politique au Nigéria, est un exemple parfait de politique culturelle visant à créer le consensus et à désamorcer les séparations et ruptures inhérentes à un espace politique vif, dynamique omniprésente dans les démocraties occidentales contemporaines tel que le montre Alain Brossat dans Le grand dégoût culturel ${ }^{15}$. L'organisation d'un contre-festival auquel sont conviés de nombreux artistes n'ayant pas été retenus dans la programmation du FESTAC est une façon pour Fela de mettre à mal l'ambition exhaustive de la version de la modernité artistique africaine proposée par le gouvernement nigérian et de donner à voir l'aspect illusoire de cette manifestation en tant qu'espace de concorde et d'entente.

24 D'autre part, l'action de Fela semble être éclairée par l'analyse foucaldienne de la « désertion-insoumission ${ }^{16}$ qui intervient lorsque l'engagement militaire est assimilé 
à une conduite politique et dans laquelle « refuser de faire le métier de la guerre ou de passer pendant un temps par cette profession et cette activité, ce refus de porter les armes apparaît comme une conduite ou une contre-conduite morale, comme un refus de l'éducation civique, comme un refus des valeurs présentées par la société, comme un refus également d'un certain rapport considéré comme obligatoire à la nation et au salut de la nation, comme un refus du rapport à la mort des autres ou du rapport à la sienne propre ${ }^{17}$. À l'occasion de son contre-festival, Fela chante Zombie devant la presse internationale et les populations des ghettos de Lagos qui en font immédiatement leur chant de protestation. Ce morceau devient l'hymne de Fela, son refus de la conduite du soldat, en tant qu'elle est l'acceptation de tous les états de fait sociétaux et politiques qu'implique son engagement militaire, et du rythme martial qui la conditionne. Cet épisode qui est tout à la fois refus du salut proposé par l'État et démonstration de stratégies de résistance et de contre-conduites possibles inquiète considérablement les autorités nigérianes qui lancent peu de temps après un assaut contre Kalakuta au cours duquel les danseuses de Afrika 70 sont violées, la mère de Fela défenestrée et ce dernier gravement blessé.

Derrière le corps du zombie désamorcé par le corps dansant de Fela, se dresse le corps de théâtre au sens où l'entend Nietzsche dans ses derniers écrits. Dans Nietzsche contre Wagner, il conceptualise deux pratiques possibles de la musique en opposant la danse comme pensée énergétique et élan vital au théâtre comme pensée démagogue. C'est une conception du théâtre comme art de la représentation dans lequel le comédien joue un rôle et se donne en spectacle, une conception qui n'en retient que l'aspect histrionique. "Personne n'apporte au théâtre le sens le plus subtil de son art, pas même l'artiste qui travaille pour le théâtre, - il y manque la solitude, tout ce qui est parfait ne tolère pas de témoins... Au théâtre, on devient peuple, troupeau, femme, pharisien, électeur, fondateur-patron, idiot - wagnérien : c'est là que la conscience la plus personnelle succombe au charme niveleur du plus grand nombre, c'est là que règne le voisin c'est là que l'on devient voisin... ${ }^{18}$. Voila semble-t-il une description assez fidèle de ce que Fela combat en dernière instance. Le soldat et l'homme du peuple sont dociles car ils ont abandonné leur puissance d'agir à un autre, à un représentant. Alors que le véritable musicien, dans la compréhension nietzschéenne, ne peut se réaliser que dans la solitude la plus complète, « dans les recoins de maisons en ruine » où " caché, caché à lui-même, il compose ses vrais chefs-d'œuvre ${ }^{19}$, c'est-à-dire dans un oubli de soi aérien, qui lui confère une supériorité, une immunité vis-à-vis de son absorption par la masse, celui qui s'adonne au théâtre accepte de se fondre dans le public, mettant ses facultés affectives et spirituelles à la merci du comédien, de celui qui convainc par la débauche d'effets spectaculaires. C'est ce corps de théâtre, qui incarne le corps du zombie aux dépens de son hôte, que Fela semble avoir voulu désarticuler en invalidant ses stratégies dont le FESTAC n'est qu'un exemple parmi d'autres.

L'autre dimension théâtrale de l'État consiste en la façon dont il met en scène sa violence et qui est théorisée par Foucault comme "pratique théâtrale de la raison d'État $»^{20}$. Par cette raison d'État il s'agit " de repérer ce qui est nécessaire et suffisant pour que l'État existe et se maintienne dans son intégrité, au besoin, si c'était nécessaire et suffisant pour rétablir cette intégrité, si elle venait à être entamée ${ }^{21}$. Cette raison d'État n'est pas un principe de transformation mais une force conservatoire qui dans la recherche de son but peut utiliser la violence, théorisée comme « coup d'État $»^{22}$, une violence théâtralisée. La raison d'État, qui « se donne elle- 
même et volontairement les lois comme cadre et comme forme $»^{23}$ devient violente lorsqu'elle devient coup d'État. "Violente, c'est-à-dire qu'elle est obligée de sacrifier, d'amputer, de faire du tort, elle est amenée à être injuste et meurtrière ${ }^{24}$. Alors que le gouvernement pastoral tel qu'il s'exprime dans une politique culturelle est une recherche de salut pour tous, le coup d'État correspond à une pastorale de l'exclusion et du sacrifice. Enfin si le coup d'État, cette violence des sages, doit s'élaborer dans le secret, sa manifestation doit être la plus éclatante possible, car elle doit signifier à la fois puissance de l'État et exalter la nécessité qui la justifie. Elle est donc mise en scène et théâtralisée. Et en effet, l'assaut contre Kalakuta que donne l'armée, quelques jours après la fin du festival, est des plus spectaculaires. Le nombre de soldats, qui s'élève à près d'un millier est totalement démesuré, le quartier est entièrement détruit, et des violences inouïes sont exercées sur tous ses habitants dont la mère de Fela défenestrée qui succombera à ses blessures quelques jours plus tard. Ces milliers de soldats que l'État nigérian ne reconnaîtra pas, à la fois corps de zombie et corps de comédiens jouant les soldats inconnus, feront l'objet d'un morceau en 1979, l'ironique Unknown Soldiers dans lequel Fela décrit la destruction de sa demeure. Ces soldats non identifiés ressemblent aux juges établis par Charlemagne pour juguler la révolte des Saxons et dont les particularités étaient d'être inconnus du public, de juger sans connaissance de cause et de juger sans procès. Pour exercer sa violence, l'État a besoin d'assassins qui tuent « qui ils veulent, comme ils veulent et sans dire pourquoi »"

Ces événements du début de l'année 1977 constituent un passage fulgurant d'une gouvernementalité de type pastoral au crime d'État, dans un souci de maintien d'intégrité étatique.

À ce passage, correspond un changement de tactique de la part de Fela qui nous fait quitter alors définitivement le monde de la musique abandonné aux ruines de Kalakuta. Dans un premier temps, il entreprend d'exhiber ses blessures et ses contusions au plus grand nombre de médias, transformant son corps amoindri matériellement en corps martyrisé, donc politique car adressant des signes forts à la communauté. Fela avait déjà utilisé cette mise en scène des humiliations subies par son corps en 1976, illustrant un disque de photos de son bras cassé et de blessures au crâne que lui avaient infligé des soldats nigérians et donnant à voir par ce procédé la brutalité aveugle de l'armée dans les stigmates de sa chair. Ensuite, assigné à résidence avant son extradition du Nigéria, comme une ultime démonstration de résistance il parvient, malgré les nombreux barrages militaires, à déposer le cercueil de sa mère devant la résidence du général Obasanjo, démentant ainsi la version officielle des soldats inconnus et préférant à la conduite du cérémonial funéraire celle de la négation d'une mort qu'il juge injuste.

29 Il nous semble que l'étude de ces quelques événements de la vie de Fela possède l'intérêt de montrer une autre voie de résistance par le corps à une époque où celle-ci se résout souvent en une simple négation de ce dernier par la destruction. Elle permet aussi de peser, par son aspect paroxystique, les périls auxquels s'exposent certaines créations artistiques en tant qu'elles constituent de profondes ruptures alors même que beaucoup d'«artistes engagés » n'ont du sauvage que la panoplie et satisfont avec zèle le besoin d'exotisme d'un vaste consensus culturel.

Enfin, nous pouvons évoquer un ultime écho de cette histoire dans le travail de Foucault autour de la notion de parrêsia. Dans Le gouvernement de soi et des autres s'élabore une conceptualisation de ce courage de la vérité et du franc-parler autour 
d'une histoire du passage de la parrêsia politique à la parrêsia philosophique. Il est dit de cette dernière qu'elle est une pratique "qui fait, dans son rapport à la politique, l'épreuve de sa réalité ", "qui trouve, dans la critique de l'illusion, du leurre, de la tromperie, de la flatterie, sa fonction de vérité », enfin "qui trouve dans la transformation du sujet par lui-même et du sujet par l'autre son objet d'exercice ${ }^{26}$. Nous reconnaîtrons volontiers au Fela des années soixante-dix l'exercice de cette pratique ambiguë, car à la fois nécessaire et dangereuse, constamment menacée par l'impuissance, l'échec, et également menace pour la vie de celui qui l'exerce ; motivée par un sentiment de devoir, accueillie comme une vertu et un talent, et nécessitant une technique, en l'occurrence musicale ; structurellement extérieure à la politique, jouant sa propre vérité dans sa confrontation à cette dernière, et privilégiant la transformation de soi et des autres à la libération.

\section{BIBLIOGRAPHIE}

A. Brossat, Le grand dégoût culturel, Paris, Seuil, 2008.

Encyclopédie Universalis, articles « Musique africaine », « Nigéria » et « Possession ».

M. Foucault, Surveiller et punir. Naissance de la prison, Paris, Gallimard, 1975.

M. Foucault, Sécurité, Territoire, Population, Paris, Gallimard/Seuil, coll. « Hautes Études », 2004.

M. Foucault, Le gouvernement de soi et des autres, t. I-II, Paris, Gallimard/Seuil, coll. « Hautes Études ", 2008.

M. K. Idowu, Fela le combattant, Le Castor Astral, 2002.

F. Mazzoleni, L'Épopée de la musique africaine. Rythmes d'Afrique atlantique,Hors Collection, 2008.

F. Nicolas, « À quel titre rapporter musique et politique », 2004, http://www.entretemps.asso.fr/ Nicolas/Textes/Politique.html

F. Nietzsche, Nietzsche contre Wagner, 1889, dans CEuvres philosophiques complètes, tome VIII, volume 1, Paris, Gallimard, 1974.

The Rough Guide to World Music, Africa \& Middle East, livre audio, World Music Network, « Rough Guide », 2007.

Lien vers la vidéo « Fela in performance (1971)» :

http://www.youtube.com/watch?v=p-SQH94Pifc\&feature=related

Lien vers le morceau Zombie par Fela Kuti et Afrika 70 :

http://www.youtube.com/watch?v=iBgewcFh-cg

\section{NOTES}

1. «Non au sexe roi » Le Nouvel Observateur, $\mathrm{n}^{\circ}$ 644, mars 1977. 
2. Michel Foucault, Sécurité, Territoire, Population, Leçon du $1^{\mathrm{er}}$ mars 1978.

3. Music is the weapon, documentaire de Stephane Tchal-Gadjieff et Jean Jacques Flori pour France Télévision, 1982.

4. Michel Foucault, Surveiller et punir, partie III « Discipline », chapitre $1^{\mathrm{er}}$ « Les corps dociles », 1975.

5. Ibid.

6. Ibid.

7. Ibid.

8. Ibid.

9. Nicole Sindzingre, article « Possession » dans Encyclopédie Universalis.

10. Ibid.

11. Music is the weapon, documentaire de Stephane Tchal-Gadjieff et Jean Jacques Flori pour France Télévision, 1982.

12. Alain Badiou dans un entretien donné au cours d'une soirée de présentation de Petit Manuel d'Inesthétique organisée par le Gtep-mp, 11 février 1999.

13. Ibid.

14. Music is the weapon, documentaire de Stephane Tchal-Gadjieff et Jean Jacques Flori pour France Télévision, 1982.

15. Alain Brossat, Le grand dégoût culturel,2008.

16. Michel Foucault, Sécurité, Territoire, Population,Leçon du $1^{\mathrm{er}}$ mars 1978.

17. Ibid.

18. Friedrich Nietzsche, Nietzsche contre Wagner, 1889.

19. Ibid.

20. Michel Foucault, Sécurité, Territoire, Population, Leçon du 15 mars 1978.

21. Ibid.

22. Ibid.

23. Ibid.

24. Ibid.

25. Ibid.

26. Michel Foucault, Le gouvernement de soi et des autres, Leçon du 9 mars 1983 - Première heure.

\section{AUTEUR}

\section{JULIEN PEREZ}

Master 2 de philosophie 